\title{
E-CADHERIN EXPRESSION IN GASTRIC ADENOCARCINOMA
}

\author{
Christopher Udayan C1, Prabhalakshmy K. K², S. Sankar ${ }^{3}$
}

1 Junior Resident, Department of Pathology, GMC, Kottayam, Kerala.

${ }^{2}$ Associate Professor, Department of Pathology, GMC, Kottayam, Kerala.

3 Professor and HOD, Department of Pathology, GMC, Kottayam, Kerala.

\section{BACKGROUND}

ABSTRACT

Gastric adenocarcinoma is one of the most common cancers in the world and 2nd most common cause of cancer deaths. It develops due to genetic and environmental factors. Histologically, Lauren's classified Gastric adenocarcinoma is classified into intestinal and diffuse types. Intestinal type can be of well differentiated, moderately differentiated or poorly differentiated. E-cadherin is a protein that helps in cell to cell adhesion.

This study is conducted to find out relation between E-cadherin expression and tumour infiltration and lymph node status.

\section{MATERIALS AND METHODS}

A descriptive study was conducted to assess E-cadherin expression in gastric adenocarcinoma specimens received in the Department of Pathology, Govt. Medical College, Kottayam during a period of 15 months (March 2016 - June 2017).

\section{RESULTS}

Among 46 cases studied, 40 (80\%) cases showed aberrant/ loss of E-cadherin expression. Among these 16 (72\%) cases were well differentiated type, $5(100 \%)$ cases were moderately differentiated, $2(100 \%)$ cases were poorly differentiated and $17(100 \%)$ cases were diffuse type. Aberrant/ loss of expression is seen in 29 (87\%) cases of lymph node positivity. Aberrant/ loss of expression is seen in 37 (88\%) cases of T3/T4 stage.

\section{CONCLUSION}

In the study aberrant E-cadherin expression was seen in 74\% cases, loss of expression in 13\% cases and normal expression in $13 \%$ cases. A negative correlation was seen between E-cadherin expression and lymph node status. Level of tumour invasion also had a negative correlation with E-cadherin expression.

\section{KEYWORDS}

E-Cadherin, Gastric Cancer, Tumour Differentiation.

HOW TO CITE THIS ARTICLE: Udayan CC, Prabhalakshmy KK, Sankar S. E-cadherin expression in gastric adenocarcinoma. J. Evolution Med. Dent. Sci. 2018;7(13):1630-1638, DOI: 10.14260/jemds/2018/369

\section{BACKGROUND}

Gastric adenocarcinoma is one of the most common cancers in the world and 2 nd most common cause of cancer deaths. ${ }^{1}$ It develops due to genetic and environmental factors. According to Borrmann's classification, stomach cancers are classified into polypoidal, fungating, ulcerative and infiltrative. $^{2}$ Histologically, Lauren classified gastric adenocarcinoma into intestinal and diffuse types. Intestinal type can be of well differentiated, moderately differentiated or poorly differentiated. ${ }^{3}$

E-cadherin is a protein that helps in cell to cell adhesion. This 120-kilodalton transmembrane glycoprotein, predominantly localised to the lateral cell border and associated with the contractile cytoskeleton, mediates homotypic adhesion and plays a key role in the organisation and maintenance of tissue structure. ${ }^{4}$ Loss of E-cadherin is associated with metastasis. Thus, blockage or downregulation of E-cadherin encoding gene ( $\mathrm{CDH} 1$ ) can be helpful in providing early detection and better prognosis.

'Financial or Other Competing Interest': None.

Submission 21-01-2018, Peer Review 10-03-2018,

Acceptance 17-03-2018, Published 26-03-2018.

Corresponding Author:

Dr. Prabhalakshmy K. K,

Njarekkattuvalappil House,

Peramangalam P. O., Thrissur-680545.

E-mail: prabha3463@gmail.com

DOI: $10.14260 /$ jemds $/ 2018 / 369$
E-cadherin expression or function is associated with changes in cellular phenotype and with the development of invasive behaviour of tumour cells, effects which can be reversed by transfection of E-cadherin-encoding cDNA. ${ }^{5}$ Evidence that down-regulation of E-cadherin can occur during tumourigenesis has been obtained in carcinogeninduced skin tumours in mice. ${ }^{6}$ Potentially, reversing methylation at E-cadherin in the gastric epithelium in patients with $\mathrm{H}$. pylori infection may halt the process of future development of gastric cancer. ${ }^{7}$ An understanding of how E-cadherin expression is regulated in normal and malignant cells is therefore a critical point in understanding tumour progression of gastric adenocarcinoma. ${ }^{8}$ This study aims in finding relation between loss of E-cadherin and gastric adenocarcinoma.

\section{Objectives of this Study are-}

1. To describe the loss/ aberrant expression of E-cadherin in gastric adenocarcinoma.

2. To describe the E-cadherin expression in various histological types of gastric adenocarcinoma.

3. To correlate between E-cadherin expression and lymph node status/ level of invasion.

\section{MATERIALS AND METHODS}

This descriptive study included first 46 cases of gastric adenocarcinoma received in Department of Pathology, Govt. Medical College, Kottayam during a period of 15 months from 
March 2016 to June 2017. The age, gender and investigation reports were collected from the clinical data sent along with the gastrectomy specimen. Cases that are proved to be gastric adenocarcinoma by histopathology report only was included for the study. Cases with differential diagnosis, small biopsy specimens was excluded. Clinical and pathological details of each cases was recorded as in the proforma.

All specimens were fixed in $10 \%$ buffered formalin. Tissue sections were taken from tumour site, processed and tissue embedded in paraffin. Sections were taken from tumour paraffin embedded blocks and stained with $\mathrm{H}$ and $\mathrm{E}$ for routine examination. IHC was performed using monoclonal mouse anti-human E-cadherin on representative gastric sections and was evaluated. Slides with normal gastric mucosa was used as positive control. Furthermore, positive E-cadherin staining in the adjacent non-involved gastric mucosa also served as an internal positive control. Immunohistochemical results were correlated with lymph node status, presence or absence of distant metastasis, size of tumour, histological type and gross type. Clinical staging for each gastric carcinoma was evaluated according to the TNM staging system indicating the extent of tumour spread. Gross appearance of the tumours was described according to the Borrmann's classification. Histomorphological tissue architecture of the tumour samples, expressed according to the Lauren's classification. E-cadherin staining was examined under a light microscope and classified according to the pattern of staining-

- E-cadherin was considered as normal when the pattern of staining was "strong and membranous."

- E-cadherin expression was considered aberrant when the pattern of staining was "Membranous and Cytoplasmic." This type of staining is also called heterogeneous. "Membranous and Faint" - this is also called homogeneous.

The E-cadherin expression is considered absent when the staining pattern was "absent." The following Statistical Methods were used to arrive at the Conclusion-

1. Ratio and proportion for age, gender, gross type, histological type and size.

2. Chi-square test for correlation of-

- Lymph node status and E-cadherin expression.

- Depth of tumour and E-cadherin expression.

For correlation purpose, the loss of E-cadherin expression and aberrant expression were together taken as aberrant. Lymph node status was divided into lymph node positive and lymph node negative. Tumour invasion, T1 and T2 stages were clubbed together and $\mathrm{T} 3$ and $\mathrm{T} 4$ stages were clubbed together.

\section{RESULTS}

The present study was conducted on 46 cases of gastric adenocarcinoma in gastrectomy specimens received in Department of Pathology, Govt. Medical College, Kottayam between March 2016 and June 2017.

\section{Age Distribution of Cases}

Among the 46 cases of gastric adenocarcinoma 16 cases (34.8\%) belonged to 50 - 59 years' age group, 12 cases (26.1\%) to $60-69$ years, 10 cases $(21.7 \%)$ to $40-49$ years and 7 cases (15.2\%) to $70-79$ years, and 1 case (2.2\%) to 30 - 39 years.

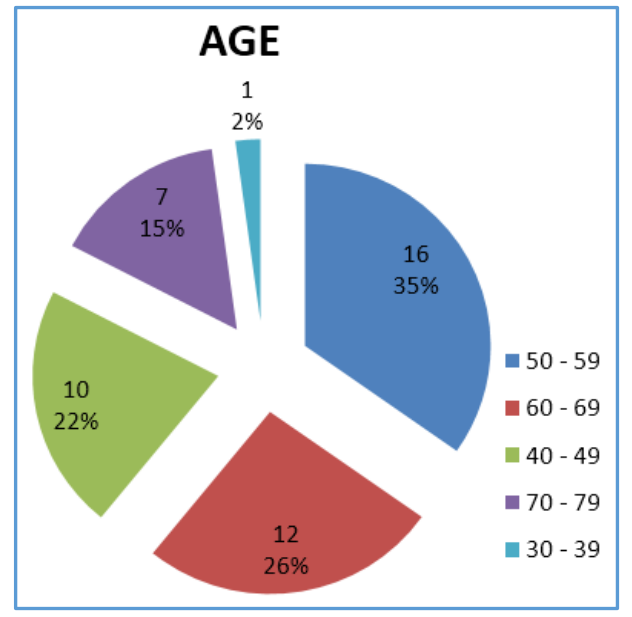

Figure 1. The Proportion of Gastric Adenocarcinoma among various Age Groups

The Distribution of Gross Types of Gastric Adenocarcinoma among various Age Groups

In all age groups, ulcerative type was predominant except in 30 - 39 age group were only one case present which was early gastric carcinoma.

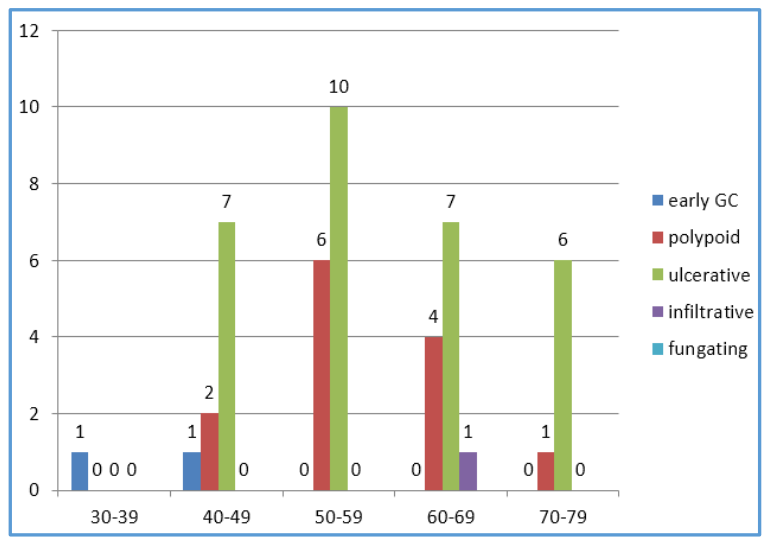

Figure 2. Distribution of Gross Types among various Age Groups

The Distribution of Histological Variants of Gastric Adenocarcinoma among various Age Groups

In the age groups $40-49$ and $50-59$, well differentiated adenocarcinoma predominates all other age group shows predominance of diffuse type.

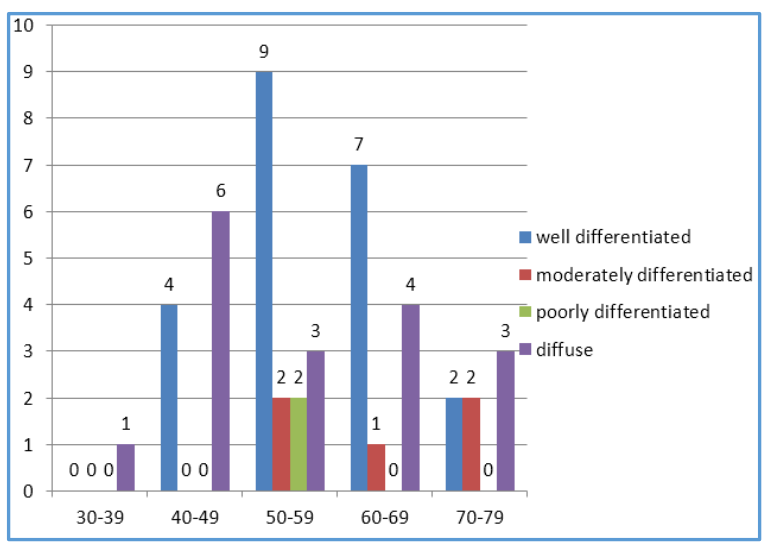

Figure 3. Distribution of Histological Types among various Age Groups 
Gender Distribution of Gastric Adenocarcinoma Cases

Among 46 cases studied, 37 cases (80\%) were males and 9 cases (20\%) were females.

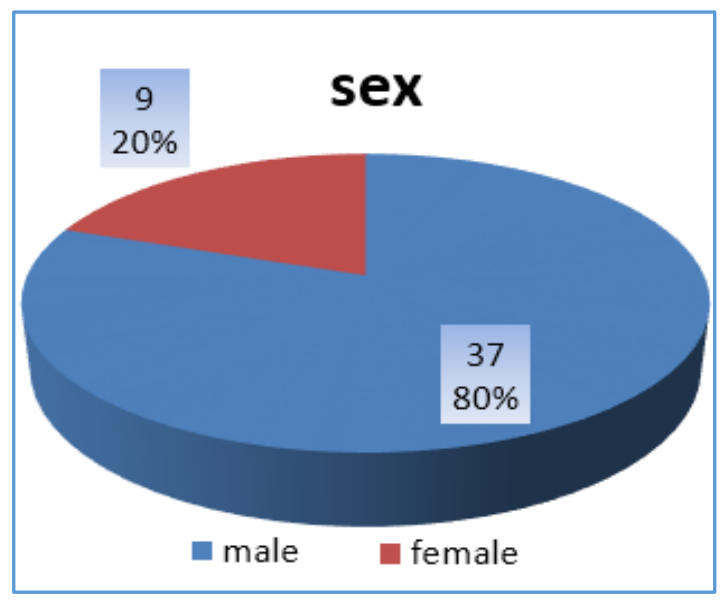

Figure 4. Gender Distribution of Cases

The Distribution of Gross Types of Gastric Adenocarcinoma among Males and Females

Among 46 cases studied, ulcerative type was predominant in both males and females.

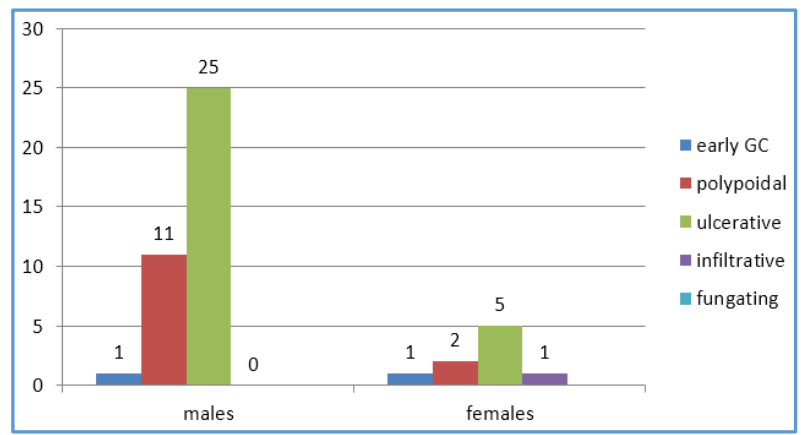

Figure 5. The Distribution of Gross Types of Gastric Adenocarcinoma among Males and Females

The Distribution of Histological Variants of Gastric Adenocarcinoma among Males and Females

In males, well-differentiated adenocarcinoma was predominant and in females diffuse type was predominant.

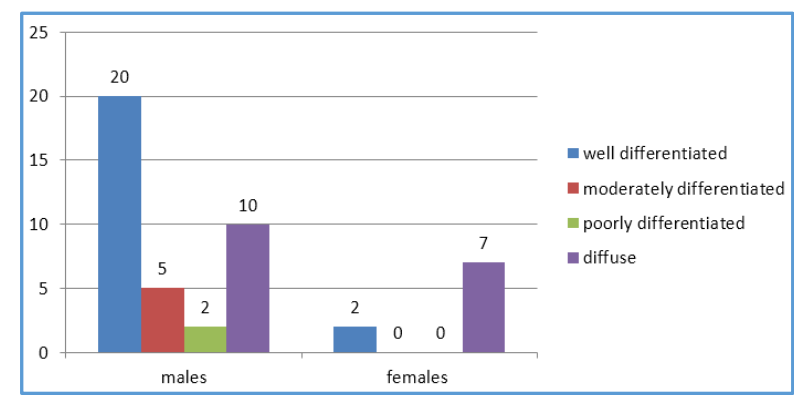

Figure 6. The Distribution of Histological Variants of Gastric Adenocarcinoma among Males and Females

\section{Gross}

Among the 46 cases collected $30(67 \%)$ cases were ulcerative type, $13(30 \%)$ cases were polypoidal type and 1 (2.2) case was infiltrative type and $2(5 \%)$ cases were early gastric carcinoma.

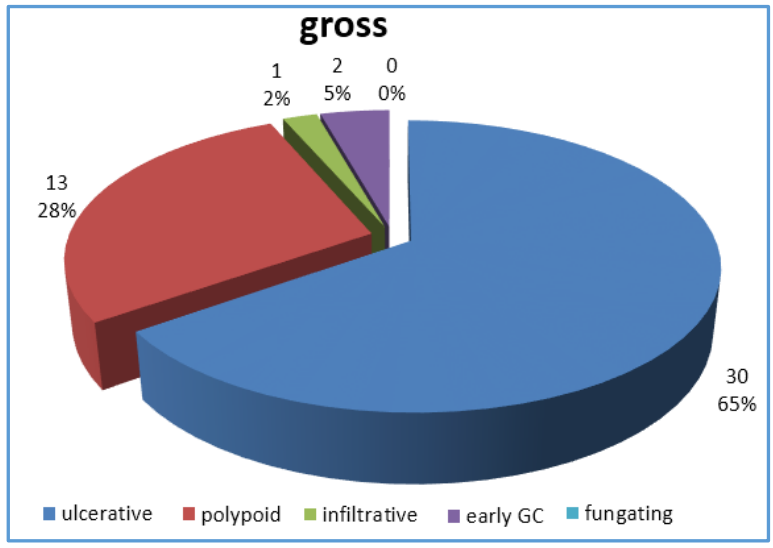

Figure 7. Distribution of Cases based on Gross Type

Distribution of Study Sample Based on Size

In the present study, 27 (59\%) cases were less than $6 \mathrm{~cm}$ and $19(41 \%)$ cases were greater than $6 \mathrm{~cm}$.

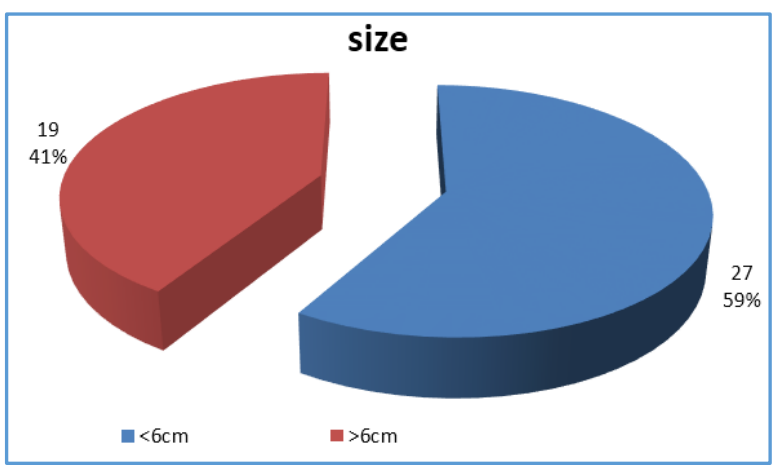

Figure 8. Distribution of Study Sample based on Size

\section{Microscopy}

Among 46 cases studied 29 (63\%) were intestinal type adenocarcinoma, of these $22(47.8 \%)$ were well differentiated adenocarcinoma, $5(10.9 \%)$ cases were moderately differentiated and 2 cases were poorly differentiated. 17 (37\%) were diffuse type.

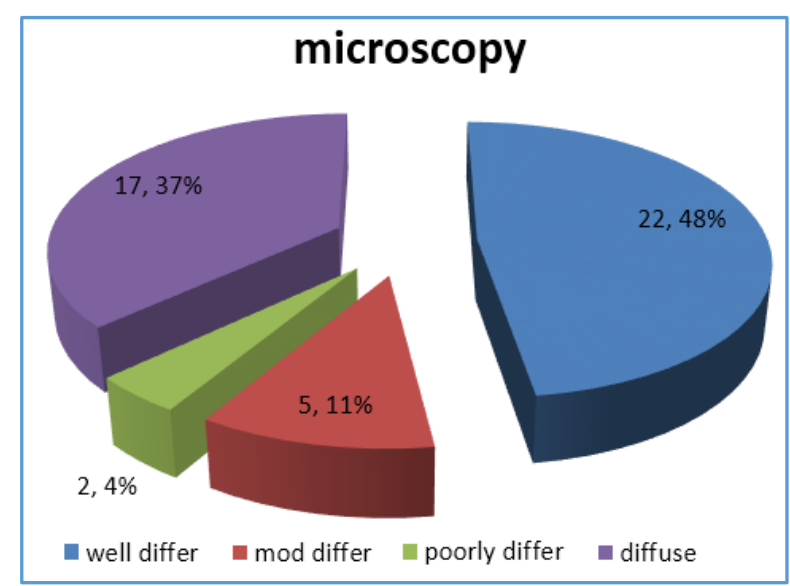

Figure 9. Distribution of Cases based on Microscopic Types

\section{Involvement of Tumour}

Among 46 cases studied, 22 (47.8\%) cases have involvement upto adjacent perigastric tissue, 20 (43.5\%) cases have involvement upto serosa, 2 (4.4\%) cases each for submucosa and muscularis propria involvement. 


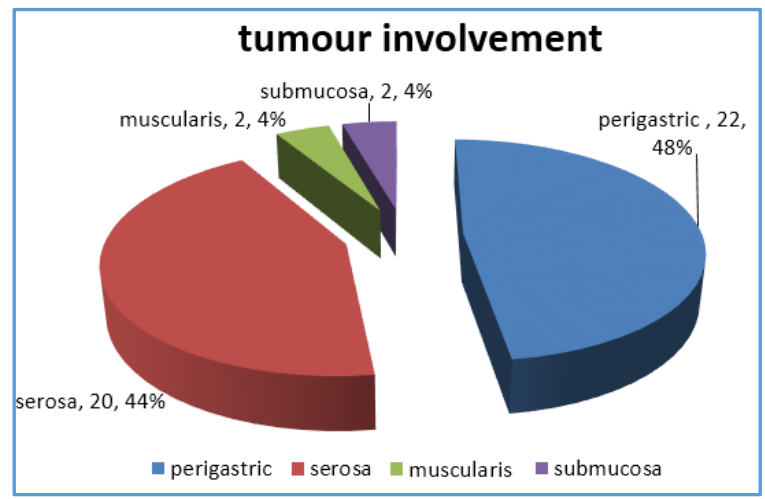

Figure 10. Distribution of Cases based on Depth of Tumour Involvement

\section{Lymph Node Status}

Among 46 cases studied $23(50 \%)$ cases had lymph nodes between 1 - 6 nodes, $8(17.4 \%)$ cases have lymph nodes between 7 - 15 and 2 (4.3\%) cases have more than 15 nodes and $13(28.3 \%)$ cases were node negative.

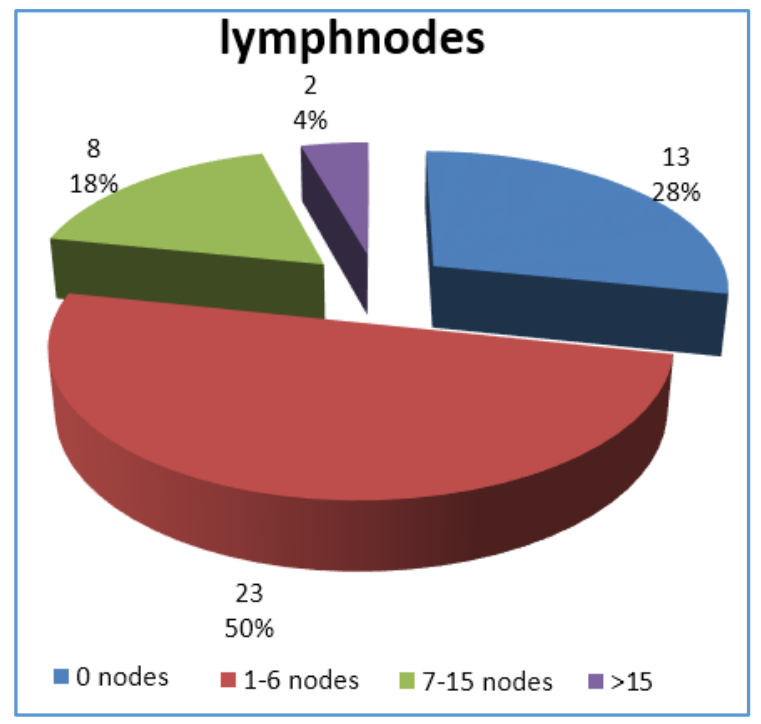

Figure 11. Distribution of Cases based on Lymph Node Status

\section{Metastasis}

Among 46 cases studied, only one case (2\%) had metastasis.

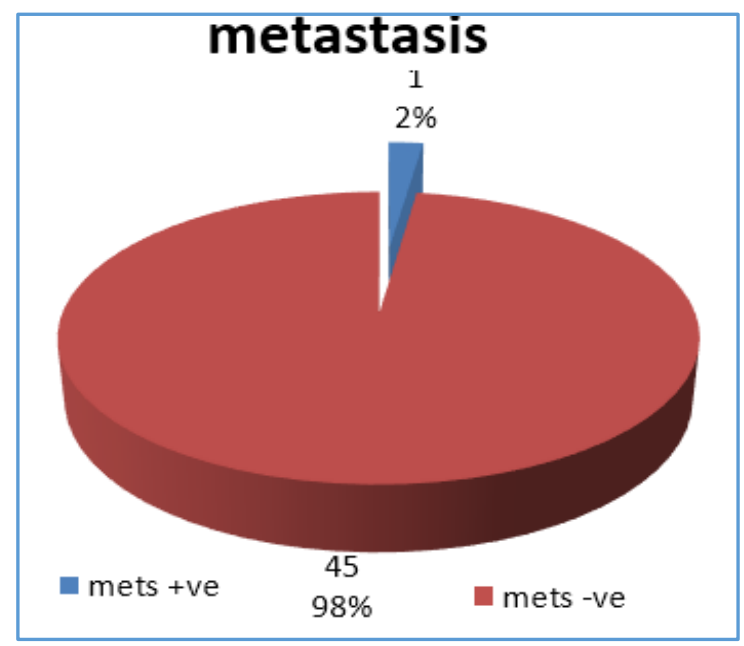

Figure 12. Distribution of Cases based on Metastasis

\section{Stage of Tumour}

Among 46 cases studied 21 (45.7\%) cases were stage 4, 13 $(28.3 \%)$ cases were stage 3a, $6(13 \%)$ cases were stage 2 and there were $2(4.4 \%)$ cases of stage $1 \mathrm{a}, 1 \mathrm{~b}$ and $3 \mathrm{~b}$ each.

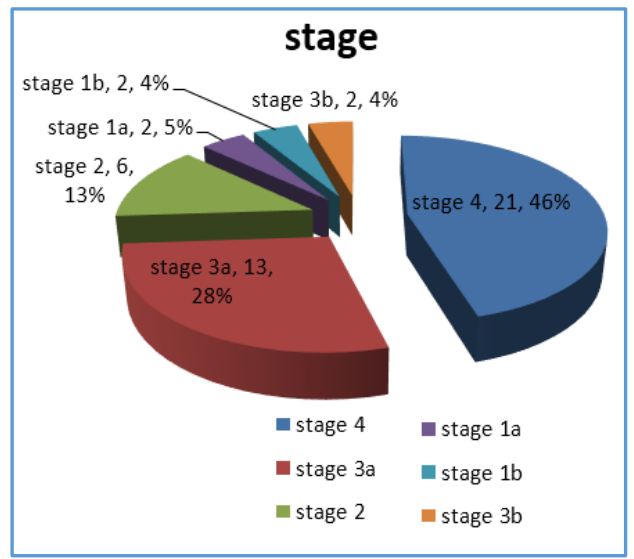

Figure 13. Distribution of Cases based on Stage of Tumour

IHC

Among 46 cases studied 34 (73.9\%) cases showed aberrant expression, $6(13 \%)$ cases showed absent expression and 6 $(13 \%)$ cases showed normal expression.

\section{e cadherin expression}

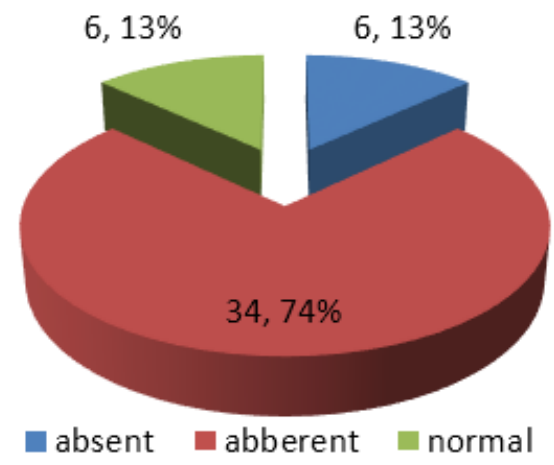

Figure 14. Distribution of Cases based on E-cadherin Expression

E-cadherin Expression among Gross Types of Gastric Adenocarcinoma

Aberrant expression was commonly seen in all gross type.

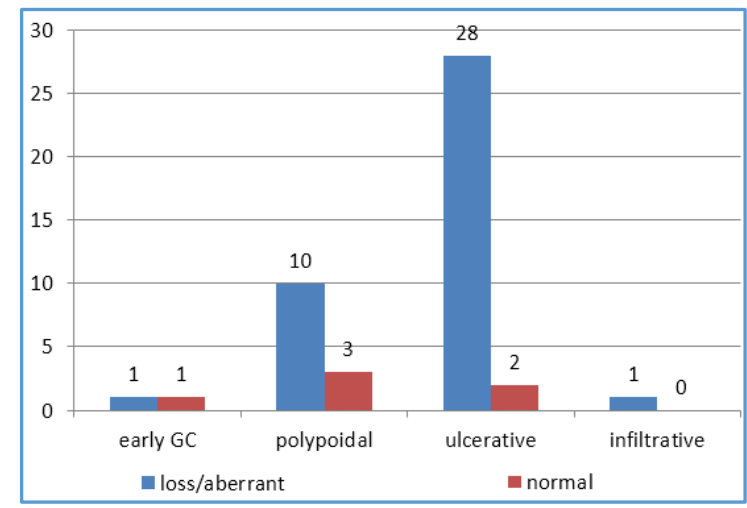

Table 15. Distribution of Study Sample based on Gross and IHC 
E-cadherin Expression Distribution based on Size

Aberrant expression was predominantly seen in all groups.

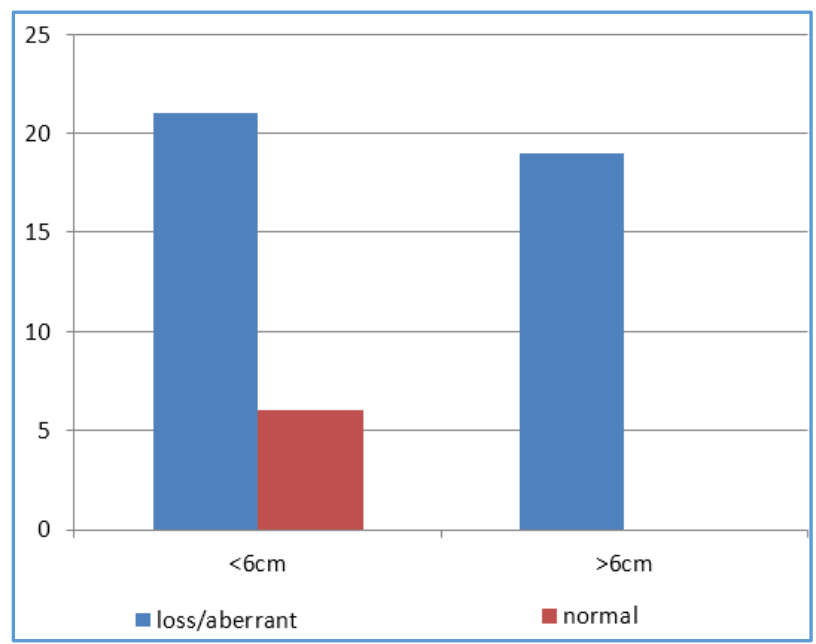

Table 16. Distribution of Study Sample based on Size and IHC Chi-Square: 4.85, P value $=0.028$ (Significant)

\begin{tabular}{|c|c|c|}
\hline & $\begin{array}{c}\text { Loss/Aberrant } \\
\text { Expression }\end{array}$ & Normal \\
\hline$<6 \mathrm{~cm}$ & $21(45 \%)$ & $6(13 \%)$ \\
\hline$>6 \mathrm{~cm}$ & $19(41 \%)$ & 0 \\
\hline \multicolumn{3}{|c|}{ Table 1. Comparison of E-cadherin Expression with } \\
Tumour Size
\end{tabular}

E-cadherin Expression among Histologic Types of Gastric Adenocarcinoma

Aberrant expression was commonly seen in all histologic type of gastric carcinoma.

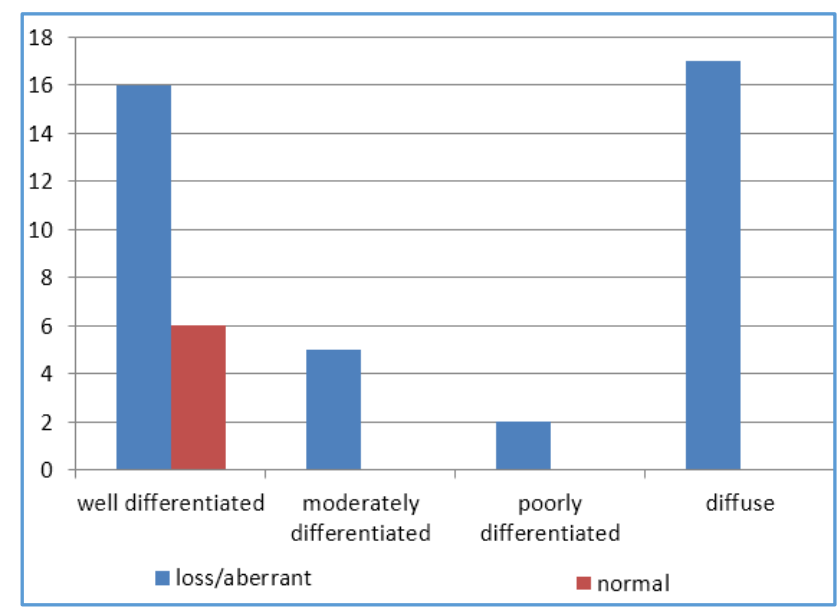

Figure 17. Distribution of Study Sample based on Microscopic Features and IHC

\begin{tabular}{|c|c|c|}
\hline & $\begin{array}{c}\text { Loss/ Aberrant } \\
\text { Expression }\end{array}$ & $\begin{array}{c}\text { Normal } \\
\text { Expression }\end{array}$ \\
\hline Well differentiated & $16(34 \%)$ & $6(13 \%)$ \\
\hline $\begin{array}{c}\text { Moderately } \\
\text { differentiated }\end{array}$ & 5 (11\%) & 0 \\
\hline $\begin{array}{c}\text { Poorly } \\
\text { differentiated }\end{array}$ & $2(4 \%)$ & 0 \\
\hline Diffuse & $17(17 \%)$ & 0 \\
\hline \multicolumn{3}{|c|}{$\begin{array}{c}\text { Table 2. Comparison of E-cadherin Expression with } \\
\text { Histological Type }\end{array}$} \\
\hline
\end{tabular}

E-cadherin Expressions Based on Depth of Tumour Invasion of Gastric Adenocarcinoma

Aberrant expression was seen commonly in all groups.

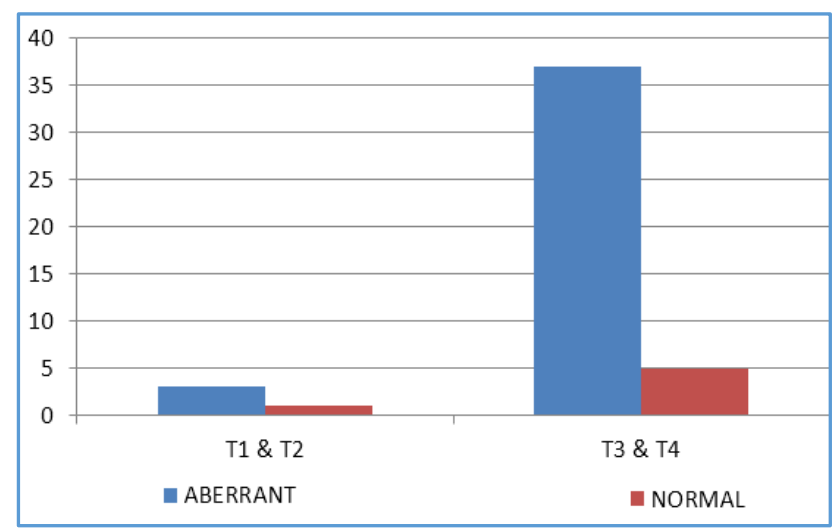

Figure 18. Distribution of Study Sample based on Depth and IHC

\begin{tabular}{|c|c|c|}
\hline & Aberrant/ Loss & Normal \\
\hline T1 and T2 & $3(6.5 \%)$ & $1(2.3 \%)$ \\
\hline T3 and T4 & $37(80 \%)$ & $5(10.8 \%)$ \\
\hline \multicolumn{3}{|c|}{ Table 3. Comparison of E-cadherin Expression with Depth } \\
of Tumour \\
\hline
\end{tabular}

Chi-square value- 0.552, P value- 0.457 (Not Significant).

Relation between Lymph Node Status and E-Cadherin Expression

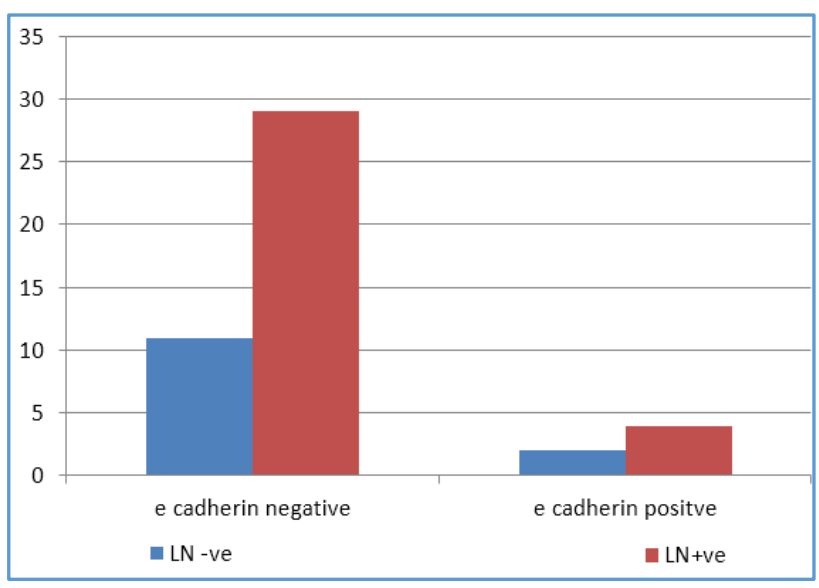

Figure 19. Distribution of Study Sample based on Lymph Node Status and IHC

Chi-square- 0.088 , P value $=.767$ (Not Significant).

\begin{tabular}{|c|c|c|}
\hline & $\begin{array}{c}\text { Lymph Node } \\
\text { Negative }\end{array}$ & $\begin{array}{c}\text { Lymph Node } \\
\text { Positive }\end{array}$ \\
\hline E-cadherin Negative & $11(23 \%)$ & $29(63 \%)$ \\
\hline E-cadherin Positive & $2(4.4 \%)$ & $4(8.6 \%)$ \\
\hline \multicolumn{2}{|c|}{ Table 4. Comparison of E-cadherin Expression with Lymph } \\
Node Status \\
\hline
\end{tabular}


E-cadherin Expression with Distant Metastasis

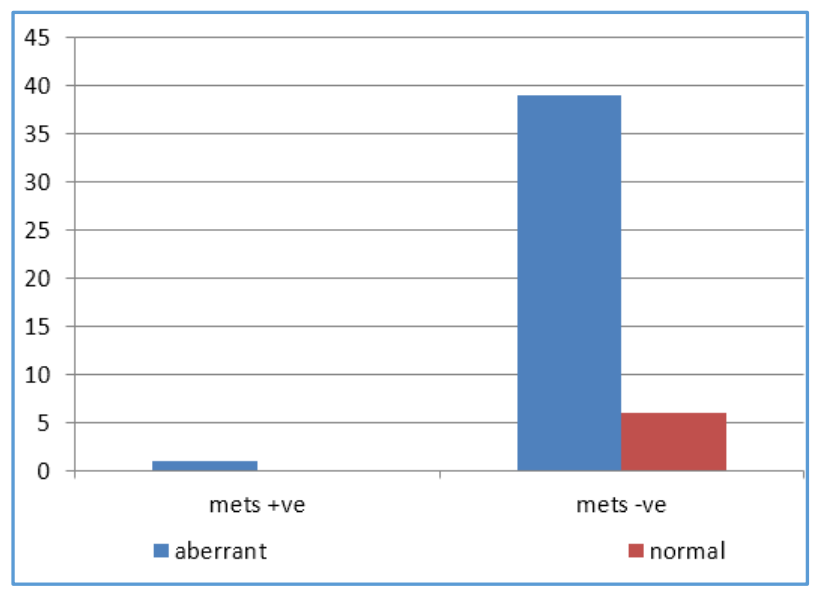

Figure 20. Distribution of Study Sample based on Mets and IHC

\begin{tabular}{|c|c|c|}
\hline & Aberrant/ Loss & Normal \\
\hline Mets Negative & 39 (84.7\%) & $6(13 \%)$ \\
\hline Mets Positive & $1(2.3 \%)$ & 0 \\
\hline Total & 40 & 6 \\
\hline \multicolumn{3}{|c|}{$\begin{array}{c}\text { Table 5. Comparison of E-cadherin Expression with Distant } \\
\text { Metastasis }\end{array}$} \\
\hline
\end{tabular}

\section{Gross Photographs}

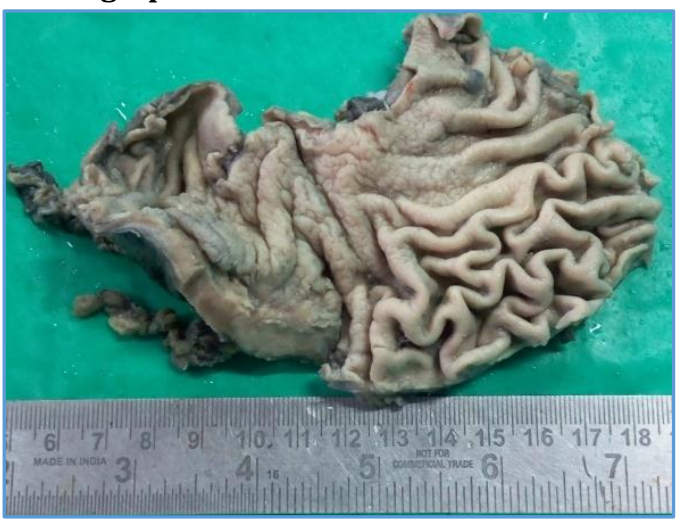

Figure 21. Gross Picture of Ulcerative Type of Gastric Adenocarcinoma

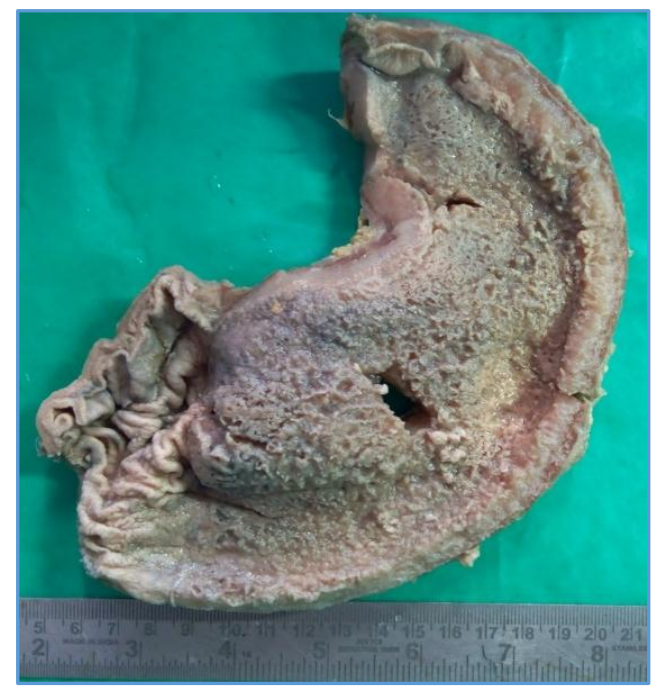

Figure 22. Gross Picture of Infiltrative Type of Gastric Adenocarcinoma

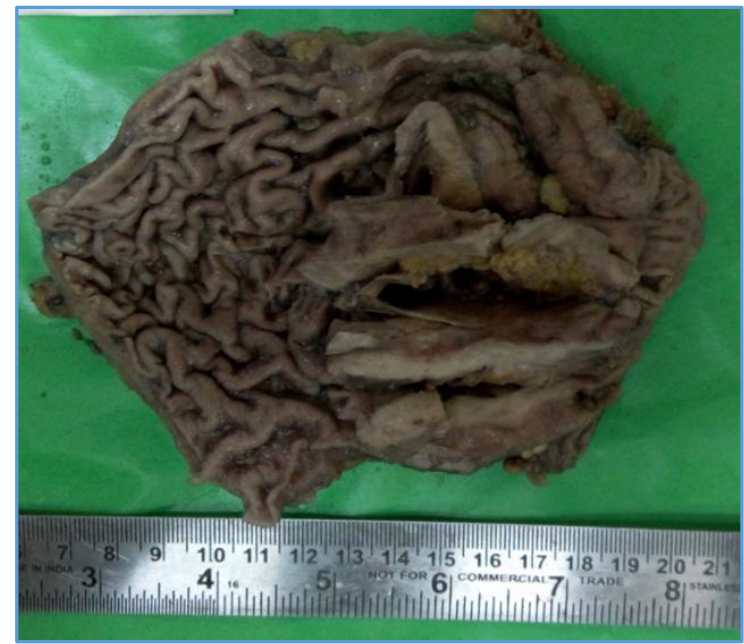

Figure 23. Gross Picture of Polypoidal type of Gastric Adenocarcinoma

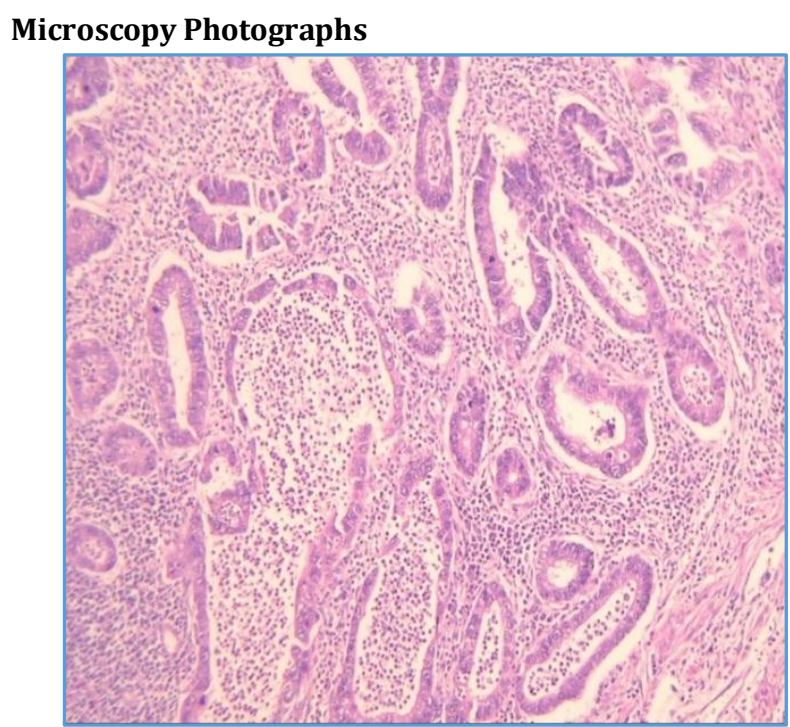

Figure 24. Photomicrograph showing Well Differentiated Gastric Adenocarcinoma (H and E, 10x)

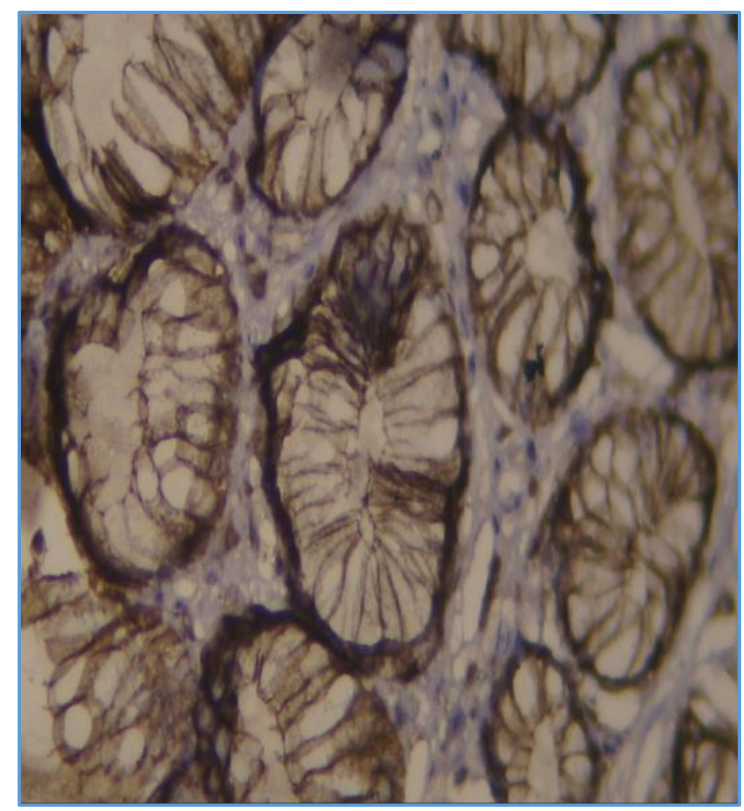

Figure 25. Photomicrograph showing Normal Expression (Strong Membrane Staining) of E-cadherin (10x) 


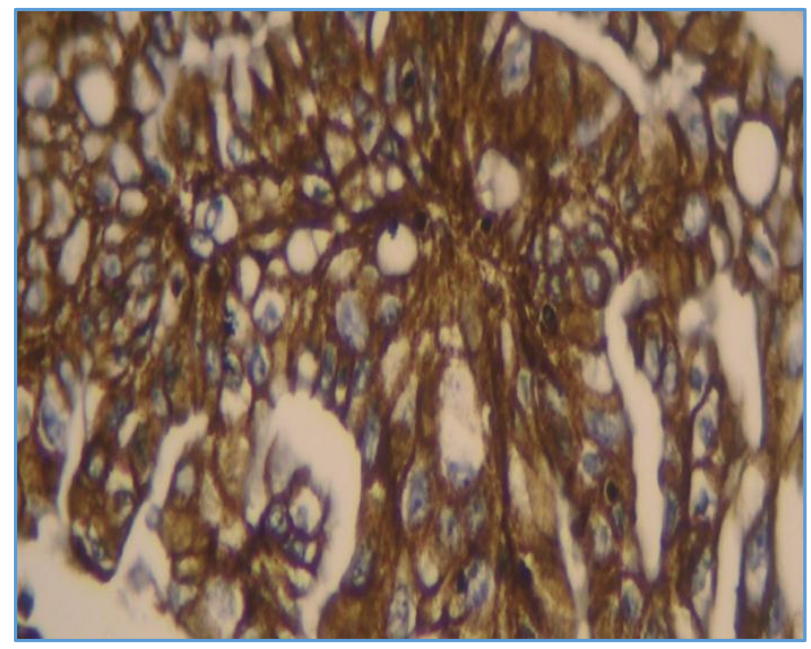

Figure 26. Photomicrograph showing Aberrant Expression (Membrane and Cytoplasmic) of E-cadherin (40x)

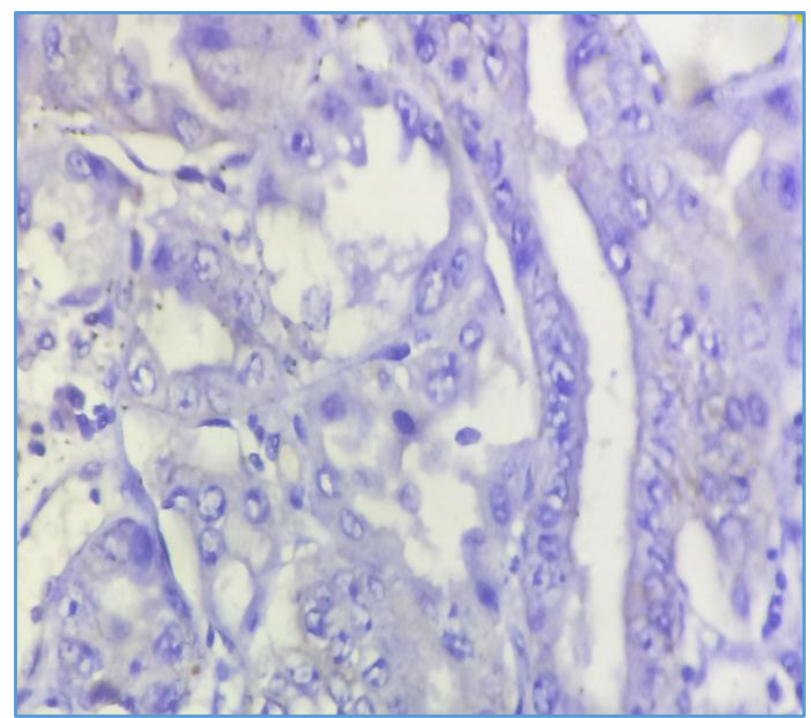

Figure 27. Photomicrograph of Loss of E-cadherin Expression in Gastric Adenocarcinoma (40x)

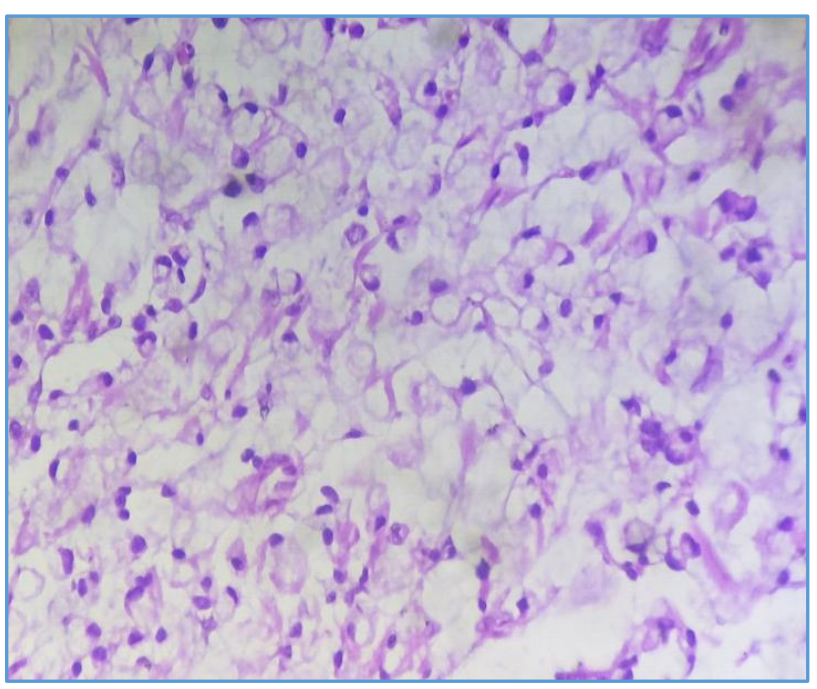

Figure 28. Photomicrograph of Diffuse Type of Gastric Adenocarcinoma (H and E, 40X)

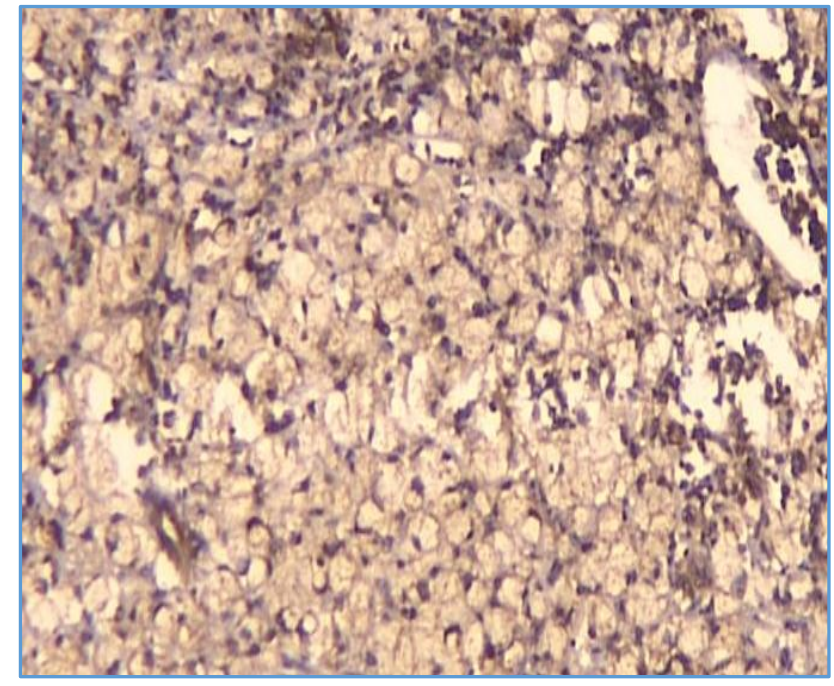

Figure 29. Photomicrograph showing Aberrant (Membrane and Cytoplasmic) Expression of E-cadherin (40x) in Diffuse Type

\section{DISCUSSION}

The present study was conducted on 46 cases of gastric adenocarcinoma specimens received in Department of Pathology, Govt. Medical College, Kottayam between March 2016 and July 2017. In the present study, the age group was ranging from 39 years to 78 years and the mean age was 56.3 years. In study conducted by Ze-yu et al, the age group was ranging from 26 years to 82 years and the mean age was 56.8 years. ${ }^{9}$ Male-to-female ratio was $4: 1$ in the present study and in study conducted by Ze-yu wu et al, the male: female ratio was 1.5: 1. In study conducted by Yong Ning Zhou et al, the age group was ranging from 28 years to 77 years and the mean age was 54.5 years. ${ }^{2}$ Male: female ratio in the study was 3.08: 1 . In study conducted by Ramesh et al ${ }^{10}$ at Department of Pathology and Surgery, Liverpool University, UK the mean age was 68 years and age range was 57 years to 87 years. Male: female ratio was 3.5: 1 . In study of 95 cases conducted by Robab Anbiaee et al, mean age was 62 years with range 19 to 85 years. ${ }^{11}$ Male-to-female ratio is $2.5: 1$.

In the present study among 46 cases 27 cases were having size less than $6 \mathrm{cms}, 19$ cases were having size greater than $6 \mathrm{cms}$. All the 19 cases greater than $6 \mathrm{~cm}$ showed aberrant/ loss of expression. There was a positive correlation between the tumour size and aberrant/ loss of expression ( $p$ value- 0.028). In the study of Robab Anbiaee et al, 46 cases were less than $5 \mathrm{~cm}$ and of that 18 showed abnormal Ecadherin expression. 48 cases were more than $5 \mathrm{~cm}$ and 33 showed loss of E-cadherin expression. In study conducted by Ze-yu wu et al, 17 cases were below $5 \mathrm{~cm}$ and 13 cases were above $5 \mathrm{~cm}$. 7 cases from each group showed loss of Ecadherin expression.

Out of the 46 cases of gastric adenocarcinoma studied 29 (63\%) intestinal type, of that 22 (48\%) were well differentiated, $5(11 \%)$ were moderately differentiated and 2 (4\%) were poorly differentiated type. In the present study, 17 (37\%) were diffuse type. Sandhyasundaram et al studied 20 cases of gastric adenocarcinoma at SRM College, Chennai where $10(50 \%)$ cases were intestinal type, in that $2(10 \%)$ cases were well differentiated, $4(20 \%)$ cases were moderately differentiated and $4(20 \%)$ cases were poorly differentiated and $10(50 \%)$ cases were diffuse type. ${ }^{3}$ 
Meyer et al studied 60 cases of gastric adenocarcinoma in University of Munich, 30 (50\%) cases were intestinal type and 21 (35\%) were diffuse type. ${ }^{4}$ Ze-yu wu et al studied 30 cases, of which 11 cases were intestinal type and 19 cases diffuse type. Ramesh et al studied 28 (70\%) cases of intestinal type and $12(30 \%)$ cases of diffuse type. Of the 28 cases of intestinal type $1(2.5 \%)$ was well differentiated, 19 (47.5\%) were moderately differentiated and 8 (20\%) cases were poorly differentiated. In study conducted by Robab Anbiaee et al 39 (41\%) cases were and 48 (59\%) cases were diffuse type.

\begin{tabular}{|c|c|c|}
\hline & Intestinal Type & Diffuse Type \\
\hline Present study & $29(63 \%)$ & $17(37 \%)$ \\
\hline Sandhyasundaram et al & $10(50 \%)$ & $10(50 \%)$ \\
\hline Meyer et al & $30(50 \%)$ & $21(35 \%)$ \\
\hline Ze-yu wu et al & $11(36 \%)$ & $19(64 \%)$ \\
\hline Ramesh et al & $28(70 \%)$ & $12(30 \%)$ \\
\hline Robabanbiaee et al & $39(41 \%)$ & $48(59 \%)$ \\
\hline Table 6. Comparison of Histological variants of Gastric \\
Adenocarcinoma Studied with other Studies \\
\hline
\end{tabular}

In all the studies including present study, intestinal type of adenocarcinoma was predominant type except in study by Ze-yu wu et al where $64 \%$ cases was diffuse.

In the present study, all of the diffuse type showed aberrant E-cadherin expression. This is in agreement with the study by Sandhyasundaram et al. Only 6 out of 29 cases in intestinal type showed normal E-cadherin expression. In study conducted by Robab Anbiaee et al, 20 out of 48 diffuse type showed aberrant E-cadherin expression. 9 out of 39 intestinal type showed aberrant expression. In study done by Ze-yu wu et al, 2 out of 11 intestinal type showed aberrant expression and 12 out of 17 diffuse type showed aberrant Ecadherin expression. In study by Sandhyasundaram et al 10 out of 10 diffuse type cases showed absent E-cadherin expression. 10 out of 10 intestinal type also showed aberrant expression.

\begin{tabular}{|c|c|c|c|c|}
\hline & \multicolumn{2}{|c|}{ Intestinal Type } & \multicolumn{2}{c|}{ Diffuse Type } \\
\cline { 2 - 5 } & Aberrant & Normal & Aberrant & Normal \\
\hline Present study & 23 & 6 & 17 & 0 \\
\hline Robabanbiaee et al & 9 & 30 & 20 & 18 \\
\hline Ze-yu wu et al & 9 & 2 & 12 & 7 \\
\hline $\begin{array}{c}\text { Sandhyasundaram } \\
\text { et al }\end{array}$ & 10 & 0 & 10 & 0 \\
\hline $\begin{array}{c}\text { Table 7. Comparison of E-cadherin Expression and } \\
\text { Histological Types with various Other Studies }\end{array}$ \\
\hline
\end{tabular}

In our present study $13(28.3 \%)$ cases were node negative, 23 (50\%) cases were in pN1 stage, 8 (17.4\%) cases were pN2 stage and $2(4.3 \%)$ cases were pN3 stage. Sandhyasundaram et al studied 20 cases of which $5(25 \%)$ cases were node negative, $5(25 \%)$ cases were in pN1 stage, $10(40 \%)$ cases were pN2 stage and $4(10 \%)$ cases pN3 stage.

In our study out of 13 node negative cases 11 cases showed aberrant staining, 2 cases showed normal membrane staining and no cases had absent staining. 6 out of 33 showed absent staining in node positive cases and 23 out of 33 showed aberrant staining. 4 out of 33 showed normal staining. This is not statistically significant. Study by Sandhyasundaram et al had 5 nodes negative cases, of which 3 showed absent staining and 6 out 15 showed absent staining in node positive cases.

\begin{tabular}{|c|c|c|c|c|}
\hline \multirow{2}{*}{} & \multicolumn{2}{|c|}{$\begin{array}{c}\text { Lymph Node } \\
\text { Positive }\end{array}$} & \multicolumn{2}{c|}{$\begin{array}{c}\text { Lymph Node } \\
\text { Negative Case }\end{array}$} \\
\cline { 2 - 5 } & $\begin{array}{c}\text { Aberrant/ } \\
\text { Loss }\end{array}$ & Normal & $\begin{array}{c}\text { Aberrant/ } \\
\text { Loss }\end{array}$ & Normal \\
\hline Present study & 29 & 4 & 11 & 2 \\
\hline $\begin{array}{c}\text { Sandhya- } \\
\text { Sundaram et al }\end{array}$ & 15 & 0 & 5 & 0 \\
\hline Ze-yu wu et al & 12 & 6 & 2 & 10 \\
\hline Yonemura et al & 47 & 14 & 19 & 12 \\
\hline Ramesh et al & 26 & 5 & 4 & 5 \\
\hline
\end{tabular}

Table 8. Comparison of E-cadherin Expression with Lymph Node Status in various Studies

Our study is in agreement with the studies of Ze-yu wu et al, Yonemura et al ${ }^{12}$ and Ramesh et al.

Study by Sandyasundaram et al showed total loss of expression in lymph node positive cases.

In our study 22 cases out of 46 were in T4 stage, 20 cases in T3 stage, 2 cases each were in $\mathrm{T} 1$ and $\mathrm{T} 2$ stages. 37 cases out of 41 cases in T3 and T4 stage showed aberrant staining and 3 out of 4 cases in T1 and T2 stage showed aberrant staining. There was no correlation between E-cadherin expression and depth of tumour invasion (p value- 0.457 ).

In study done by Sandhyasundaram et al, 15 cases were in $\mathrm{T} 3$ and $\mathrm{T} 4$ stage and 5 cases were in $\mathrm{T} 1$ and $\mathrm{T} 2$ stage. All cases showed aberrant staining. In study conducted by Robabanbiaee et al, 34 out of 52 cases in T3 and T4 stage showed aberrant staining.

Our study is in agreement with the study by Robab Anbiaee et al.

\begin{tabular}{|c|c|c|c|c|}
\hline & \multicolumn{2}{|c|}{ T1 and T2 } & \multicolumn{2}{c|}{ T3 and T4 } \\
\cline { 2 - 5 } & Aberrant & Normal & Aberrant & Normal \\
\hline Present study & 3 & 1 & 37 & 5 \\
\hline $\begin{array}{c}\text { Sandhyasundaram } \\
\text { et al }\end{array}$ & 5 & 0 & 15 & 0 \\
\hline Robabanbiaee et al & 28 & 15 & 34 & 18 \\
\hline Table 9. Comparison of E-cadherin Expression with \\
Tumour Depth in various Studies \\
\hline
\end{tabular}

\section{Limitation of this Study}

Small sample size and short duration of the study are the limitations. Multivariate analysis with a larger sample size and long-term follow-up of the cases only can assess the utility of E-cadherin expression loss in predicting the outcome of the disease.

\section{CONCLUSION}

The present study was done on 46 cases of gastric adenocarcinoma. Aberrant E-cadherin expression was seen in $74 \%$ cases, loss of expression in $13 \%$ cases and normal expression in $13 \%$ cases.

Among 29 cases of intestinal type aberrant/ loss of expression is seen in $72.7 \%$ of well differentiated type, $100 \%$ cases of moderately differentiated and $100 \%$ cases of poorly differentiated type. Normal expression was seen in $27.3 \%$ cases of well differentiated type. 100\% cases of diffuse type also showed aberrant/ loss of expression.

In this study, a negative correlation was seen between Ecadherin expression and lymph node status. Level of tumour invasion also had a negative correlation with E-cadherin expression. 


\section{REFERENCES}

[1] Dewan K, Madan R, Sengupta P, et al. Analysis of epithelial-cadherin and human epidermal growth factor receptor 2/expression in gastric carcinoma using immunohistochemistry. Indian Journal of Pathology and Microbiology 2015;58(2):154-7.

[2] Zhou YN, Xu CP, Han B, et al. Expression of E-cadherin and $\beta$-catenin in gastric carcinoma and its correlation with the clinicopathological features and patient survival. World Journal of Gastroenterology 2002;8(6):987-93.

[3] Sundaram S, Rao S, Prathiba D. E-cadherin expression: a signature for invasion in gastric cancer. SRJM 2010;3(1):14-17.

[4] Mayer B, Johnson JP, Leitl F, et al. E-cadherin expression in primary and metastatic gastric cancer: down-regulation correlates with cellular dedifferentiation and glandular disintegration. Cancer Research 1993;53(7):1690-5.

[5] Takeichi M. Cadherins: a molecular family important in selective cell-cell adhesion. Annual review of Biochemistry 1990;59(1):237-52.

[6] Navarro P, Gómez M, Pizarro A, et al. A role for the Ecadherin cell-cell adhesion molecule during tumor progression of mouse epidermal carcinogenesis. The Journal of Cell Biology 1991;115(2):517-33.
[7] Sun GY, Wu JX, Wu JS, et al. Caveolin-1, E-cadherin and $\beta$-catenin in gastric carcinoma, precancerous tissues and chronic non-atrophic gastritis. Chinese Journal of Cancer Research 2012;24(1):23-8.

[8] Behrens J, Löwrick 0, Klein-Hitpass L, et al. The Ecadherin promoter: functional analysis of a G.C-rich region and an epithelial cell-specific palindromic regulatory element. Proceedings of the National Academy of Sciences USA 1991;88(24):11495-9.

[9] Wu ZY, Zhan WH, Li JH, et al. Expression of E-cadherin in gastric carcinoma and its correlation with lymph node micrometastasis. World Journal of Gastroenterology WJG 2005;11(20):3139-43.

[10] Ramesh S, Nash J, McCulloch PG. Reduction in membranous expression of beta-catenin and increased cytoplasmic E-cadherin expression predict poor survival in gastric cancer. British Journal of Cancer 1999;81(8):1392-7.

[11] Anbiaee R, Sheibani KM, Torbati P, et al. Abnormal expression of E-cadherin in gastric adenocarcinoma and its correlation with tumor histopathology and helicobacter pylori infection. Iranian Red Crescent Medical Journal 2013;15(3):218-22.

[12] Yonemura Y, Endou Y, Kimura K, et al. Inverse expression of S100A4 and E-cadherin is associated with metastatic potential in gastric cancer. Clinical Cancer Research 2000;6(11):4234-42. 